\title{
(2) OPEN ACCESS \\ Five-year follow-up of new cases after a coeliac disease mass screening
}

\author{
Olof Sandström 자, ${ }_{1}^{1}$ Fredrik Norström ${ }_{1}{ }^{2}$ Annelie Carlsson, ${ }^{3}$ Lotta Högberg, ${ }_{1}{ }^{4}$ \\ Maria van der Palz, ${ }^{3}$ Lars Stenhammar, ${ }^{4}$ Charlotta Webb, ${ }_{1}^{3}$ Anneli Ivarsson, ${ }_{1}^{2}$ \\ Anna Myléus (10 ${ }^{5}$
}

- Additional supplemental material is published online only. To view, please visit the journal online (http://dx.doi. org/10.1136/archdischild2021-322755)

${ }^{1}$ Department of Clinical Sciences, Paediatrics, Umea University, Umea, Sweden ${ }^{2}$ Department of Epidemiology and Global Health, Umea University, Umea, Sweden ${ }^{3}$ Department of Clinical Sciences, Paediatrics, Lund University, Lund, Sweden ${ }^{4} 4$ Department of Clinical and Experimental Medicine, Paediatrics, Linköping University, Linkoping, Östergötland, Sweden

${ }^{5}$ Department of Public Health and Clinical Medicine, Family Medicine, Umea University, Umea, Sweden

\section{Correspondence to}

Dr Olof Sandström, Department of Clinical Sciences, Paediatrics, Umea University, Umea 90187, Sweden;

olof.sandstrom@umu.se

Received 29 June 2021

Accepted 28 November 2021

\begin{abstract}
Objective We previously performed a population-based mass screening of coeliac disease in children aged 12 years in two birth cohorts resulting in 296 seropositive children, of whom 242 were diagnosed with coeliac disease after duodenal biopsies. In this follow-up study, we wanted to identify new cases in the screening population that tested negative-either converting from potential coeliac disease (seropositive but normal duodenal mucosa) or converting from seronegative at screening to diagnosed coeliac disease.

Methods All seropositive children were invited to a follow-up appointment 5 years after the screening with renewed serological testing and recommended endoscopic investigation if seropositive. Seronegative children in the screening study $(n=12353)$ were linked to the National Swedish Childhood Coeliac Disease Register to find cases diagnosed in healthcare during the same period.
\end{abstract}

Results In total, 230 (77\%) came to the follow-up appointment, including 34 of 39 with potential coeliac disease. Of these, 11 (32\%) had converted to coeliac disease. One new case was found in the National Swedish Childhood Coeliac Disease Register who received the diagnosis through routine screening in children with type 1 diabetes.

Conclusions There is a high risk of conversion to coeliac disease among those with potential disease. However, a negative screening test was associated with a very low risk for a clinical diagnosis within a follow-up period of 5 years.

\section{INTRODUCTION}

Coeliac disease is an immune-mediated enteropathy characterised by inflammatory lesions in the small intestine triggered by dietary gluten in genetically susceptible individuals. ${ }^{1}$ In children, the disease is diagnosed through verification of the enteropathy by a small intestinal biopsy or repeated serology if the criteria for the non-biopsy approach are fulfilled. It is well known that coeliac disease can cause gastrointestinal symptoms, growth retardation and anaemia. However, symptoms can be very vague and challenging to recognise in clinical practice. ${ }^{1}$ From screening studies, we know that the disease is highly underdiagnosed in both children and adults. ${ }^{2-6}$ We have previously shown that active case findings extended to the general population would be ineffective and only population mass screening can identify most coeliac disease cases. ${ }^{7}$

\section{What is already known on this topic?}

In a clinical context, there is a high risk of developing coeliac disease in children who have tested seropositive but who have normal mucosal architecture.

- Mass screening is needed to identify most coeliac disease cases in children.

\section{What this study adds?}

In a screening context, potential coeliac disease implies a high risk of conversion to disease with enteropathy within 5 years from a screening and needs to be followed up.

- The risk of receiving a clinical diagnosis within 5 years from a screening is very low.

- A possible repeated screening can wait longer than 5 years.

Undiagnosed and untreated coeliac disease, even in asymptomatic cases, can have negative health consequences in the long run related to malabsorption and increased risk of intestinal malignancies. ${ }^{8-10}$ However, the natural history of coeliac disease is not completely understood. ${ }^{11}$ Repeated screening of risk groups, that is, individuals with type 1 diabetes, Down syndrome, Turner syndrome or having a first-degree relative with coeliac disease, are recommended. ${ }^{1}$ An unanswered question is if a single screening event in the general population is enough or whether a possible mass screening must be repeated because coeliac disease can develop anytime during the lifespan. ${ }^{12}$

In 2005-2006 and 2009-2010, we performed a school-based coeliac disease mass screening of children aged 12 years. ${ }^{613}$ We found a coeliac disease prevalence of 29/1000 and 22/1000, respectively, with two-thirds undiagnosed prior to the screening in both phases. Additionally, we identified a proportion of children with potential coeliac disease in both birth cohorts. Potential coeliac disease refers to a positive serological test but with normal duodenal mucosal architecture on small intestinal biopsy, and it is generally not treated with a gluten-free diet if asymptomatic. ${ }^{1}$

The aim of this study was to identify new cases of coeliac disease in the screening population during a 5 -year follow-up period, including both cases that 
converted from potential coeliac disease to coeliac disease and clinically diagnosed cases among children who were seronegative at the time of the screening.

\section{METHOD}

\section{Overall study design}

We conducted a 5-year follow-up after a population-based mass screening in two birth cohorts of children. We followed two groups with different approaches: (1) children with positive serology in the screening were invited to a follow-up appointment at the paediatric clinic and (2) the group of children with negative (normal) serology were linked to the National Swedish Childhood Coeliac Disease Register to identify any new cases of coeliac disease diagnosis.

\section{Information on the preceding screening study}

Details of the screening study Exploring the Iceberg of Coeliacs in Sweden (ETICS) have been previously published. ${ }^{6}{ }^{13} 14$ In total, we invited 18325 children aged 12 years with 13279 consenting to participate, including 100 children with coeliac disease diagnosed prior to the study. Levels of antitissue transglutaminase IgA and IgG antibodies (anti-TG2-IgA and $\operatorname{IgG}$ ) were measured by ELISA (Celikey Phadia, Freiburg, Germany). The recommended cut-off for the test was $5 \mathrm{U} / \mathrm{mL}$, but we used a reduced level to increase sensitivity. Positive serology was defined as anti-TG2-IgA $>4 \mathrm{U} / \mathrm{mL}$, or intermediate levels of anti-TG2 IgA $(2-4 \mathrm{U} / \mathrm{mL})$ in combination with endomysial antibody (EMA) positivity of 1:5 or greater. Blood samples were obtained from 12632 children, whereof 296 tested positive and were recommended to undergo small intestinal biopsies. Biopsies were evaluated by local pathologists, and an expert pathologist re-evaluated all specimens blinded to the results of the local pathologists' assessments. ${ }^{6}$

In the first screening phase (2005-2006), 182 children accepted a small intestinal biopsy resulting in 153 new cases of coeliac disease. In the second screening phase (2009-2010), 99 children accepted intestinal biopsy with 89 new cases. Children with a biopsy showing normal histopathology without symptoms or signs typical for coeliac disease were classified as potential coeliac disease, including 29 cases in the first screening and 10 in the second screening (figure 1). In total, 12353 children had negative serology in the screening.

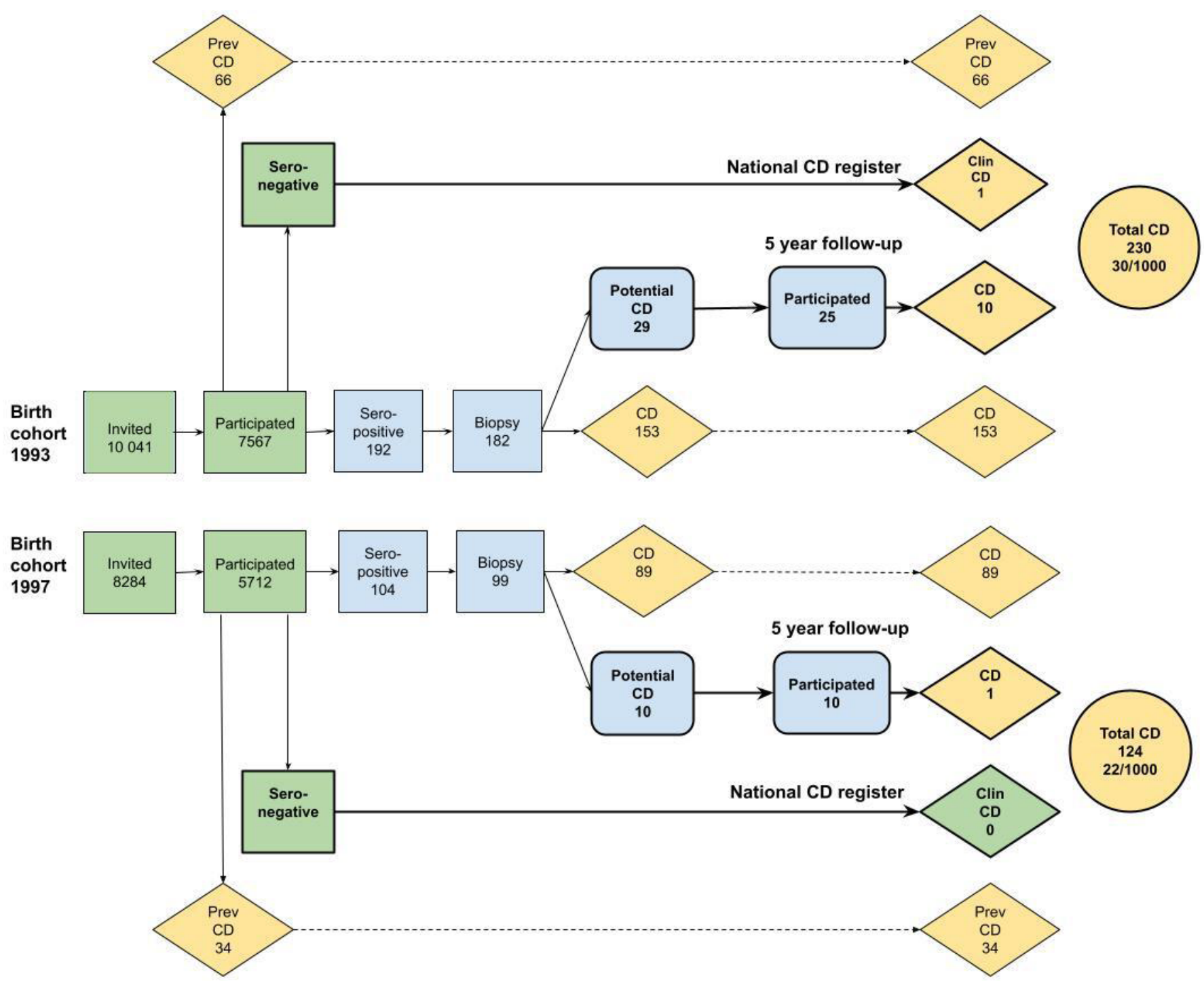

Figure 1 Screening for coeliac disease (CD) in two phases and 5-year follow-up of both children with positive serology (clinical appointment) and negative serology (register based) summarising total number of CD cases in two birth cohorts. 


\section{Five-year follow-up appointment}

Five years after the screening, in 2011 and 2015, for the first and the second screening phases, respectively, we invited all children with positive serology for a clinical follow-up at the local paediatric clinic responsible for the ETICS study. Children with potential coeliac disease were recommended endoscopic small intestinal biopsy if serology remained positive. This clinical decision was based on the local analysis of anti-TG2-IgA.

Small intestinal biopsies obtained by endoscopy were evaluated by a local pathologist.

\section{Follow-up using the National Swedish Childhood Coeliac Disease Register}

To assess the risk for a clinical diagnosis of coeliac disease among the children with negative serology in the screening, we linked the group $(n=12353)$ to the 'National Swedish Childhood Coeliac Disease Register'. ${ }^{15}$ The register is a nationwide prospective incidence register. All paediatric clinics performing small intestinal biopsies report new cases, mostly with full personal identity number allowing linkage on the individual level to other data sources. ${ }^{16}$ The register data have undergone quality assessment for the period 2010-2017 (unpublished data).

\section{Statistical analyses}

Data handling and statistical analyses were performed with Access 2016 (Microsoft, Redmond, Washington, USA). Descriptive statistics were used, and proportions were compared with the $\chi^{2}$ test or Fisher's exact test. IBM SPSS V.26 2019 was used to compare means with two-way analysis of variance. Statistical significance was defined as $\mathrm{p}<0.05$.

\section{RESULTS}

Follow-up of children with positive serology

Of the 296 invited children, 230 (77\%) came to the follow-up appointment at the paediatric clinic.

Twenty-four of 29 children with potential coeliac disease from the first screening phase, and all 10 potential cases from the second screening phase participated in the 5-year follow-up. Ten out of 24 from the first phase and 1 out of 10 from the second were diagnosed with coeliac disease rendering 11 new cases (figure 1), all confirmed with small intestinal biopsies regardless of level of anti-TG2-IgA. Details of the children with potential coeliac disease who had converted to disease are presented in table 1. Sex and level of anti-TG2-IgA at screening did not differ between the converting and non-converting group. EMA positivity was more common in the converting group (online supplemental table 1). More cases of potential coeliac disease were found in the first screening compared with the second $(29(0.38 \%)$ vs $10(0.18 \%) ; \mathrm{p}=0.0012)$. The proportion converting to coeliac disease was also higher, although not statistically significant (10 out of 29 (34\%) vs 1 out of $10(10 \%) p=0.245)$.

\section{Follow-up of children with negative serology}

Through linking the group of children with negative serology $(\mathrm{n}=12353)$ to the National Swedish Childhood Coeliac Register, we identified one child from the first phase who was negative for anti-TG2-IgA at the screening but had been reported to the register with a coeliac disease diagnosis before the 5 -year follow-up. This child had a diagnosis of type 1 diabetes and had been screened annually in line with prevailing guidelines. No new cases from the second phase were found (figure 1).

Table 1 Characteristics of children diagnosed with coeliac disease during the 5-year follow-up

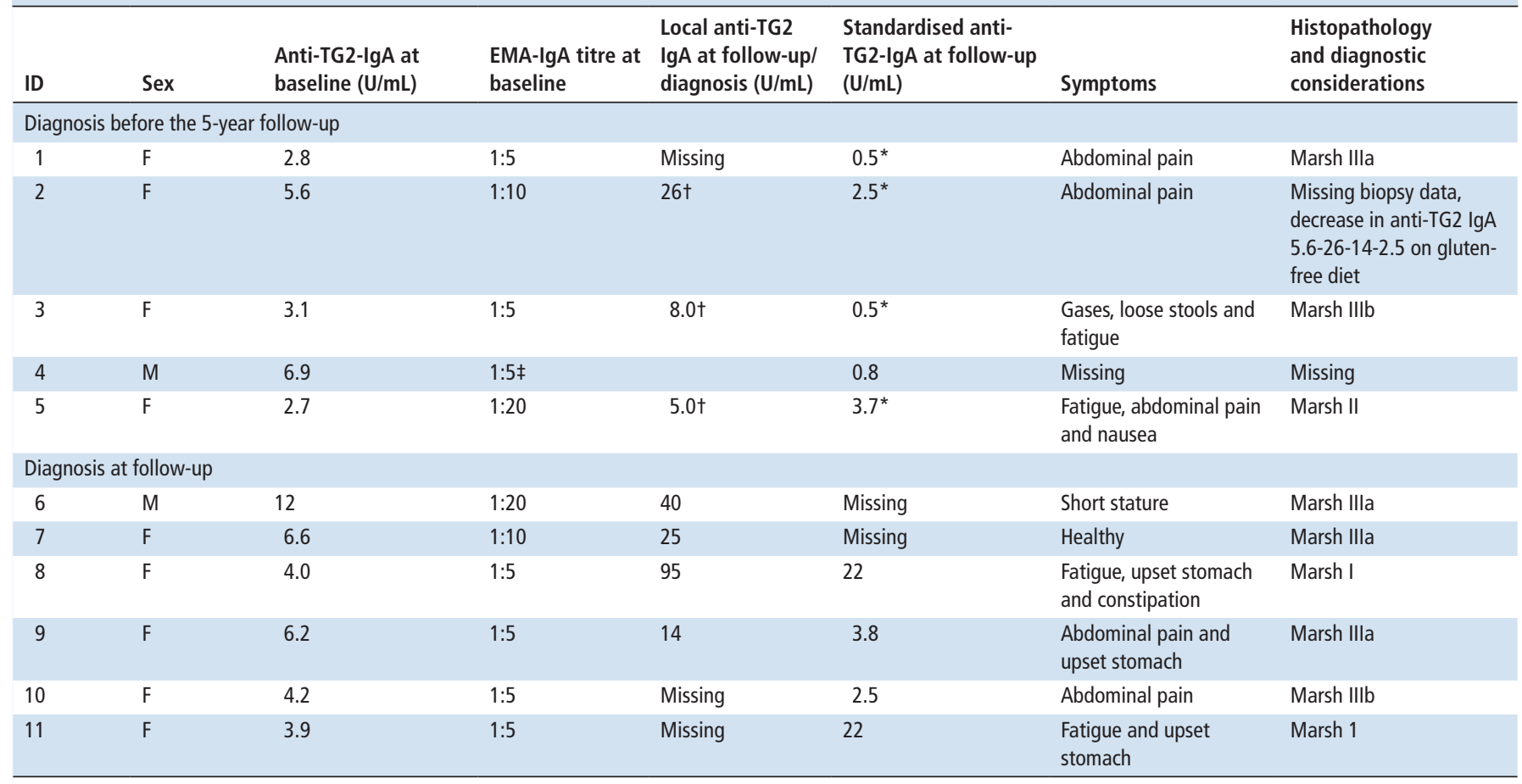

${ }^{*}$ At 5-year follow-up on a gluten-free diet.

tAt diagnosis, before the 5-year follow-up.

\#EMA IgG and EMA IgA neg.

EMA, endomysial antibody. 


\section{DISCUSSION}

In this study, we report that children with potential coeliac disease, that is, those who are seropositive but with normal small intestinal biopsies, are at high risk of conversion to coeliac disease during the following 5 years. However, in this age group, a negative screening test was associated with a very low risk for a clinical coeliac disease diagnosis during the same period.

The ETICS study was the first large population-based coeliac disease screening in children in Sweden. ${ }^{13}$ We showed that although active case finding is recommended, and screening of risk groups is implemented, most cases with coeliac disease remain undiagnosed. ${ }^{67}$ In our opinion, a population-based screening is the only way to find most coeliac disease cases. Whether mass screening for coeliac disease should be introduced is a complex question under debate. ${ }^{11} 1718$ We have previously shown that the performance of our screening strategy worked well with an estimated positive predictive value of $80 \%$ even though we used a reduced cut-off for anti-TG2-IgA. ${ }^{14}$ In 2012, the European Society for Paediatric Gastroenterology Hepatology and Nutrition (ESPGHAN) introduced the possibility of a no-biopsy approach for diagnosing symptomatic children if anti-TG2-IgA is 10 times above the normal upper limit for the specific test. In our material, we showed that the same cutoff for a no-biopsy approach could also be used in a screening situation. ${ }^{19}$ In 2020, ESPGHAN revised their guidelines, and the no-biopsy now also applies to asymptomatic children. Applying these guidelines to the follow-up, one child could have been diagnosed without the biopsy. In a recently published health economic evaluation, we showed that coeliac disease screening of children aged 12 years can be considered cost effective, especially if the children diagnosed adhere to a gluten-free diet. ${ }^{20}$ Of the children in the second phase of the screening, 69\% reported to be always gluten free and $25 \%$ often gluten free at the 5 -year follow-up. ${ }^{21}$ Corresponding numbers for the first phase were $56 \%$ and $29 \%$ for always gluten free and often gluten free, respectively (unpublished data).

One additional important question concerns the natural history of coeliac disease. In this study, we had the possibility to follow 39 cases of potential coeliac disease for 5 years and found that about one-third developed coeliac disease within this period. However, it is notable that there was a considerable difference between the two birth cohorts. The first screening phase included the birth cohort of 1993, born during a coeliac disease epidemic and they had significantly higher prevalence also at the follow-up compared with the second phase of the study when the 1997 birth cohort was screened. ${ }^{6}$ We cannot exclude that the environmental factors increasing the risk for coeliac disease in the 1993 cohort also affected the risk for potential coeliac disease and conversion to coeliac disease. In line with the findings from our 1997 cohort, Volta et al ${ }^{22}$ followed 16 asymptomatic adults with potential coeliac disease over 3 years and only one converted. Auricchio et al followed cases over a longer period (12 years) and found that $43 \%$ of 280 potential cases developed coeliac disease. ${ }^{23}$ A limitation of our study was that the number of potential coeliac disease cases was relatively low, and not everyone participated in the follow-up, making our prevalence estimates somewhat underestimated. We relied on local routine diagnostic assessments, but serological findings were verified with a second analysis for the respective cohort. We conclude that children who have tested positive to anti-TG2-IgA at one time point need to be followed for a long period because of high risk of developing coeliac disease later in life.
Because coeliac disease can present at any time during the life span, another important question is whether a mass screening must be repeated and, in that case, how often. When we followed up the seronegative children through linkage between the coeliac disease mass screening study and the National Swedish Childhood Coeliac Disease Register, we could only identify one new case of coeliac disease 5 years later. This child belonged to a well-known risk group who are screened annually for coeliac disease. In the latter cohort, we did not find any new cases. This indicates that if testing negative for anti-TG2-IgA the risk of being diagnosed with coeliac disease through routine clinical practice within 5 years after a mass screening is very low in this age group. However, there are some limitations to this conclusion. We know that most coeliac disease cases are reported to the National Swedish Childhood Coeliac Disease Register but not all. Thus, we cannot exclude that we missed some children. Only diagnosed cases are reported to the register, meaning that those who were overlooked in clinical care and who were never tested again because they had negative serology in the study or were truly asymptomatic would be missed. This could contribute to an underestimation of new coeliac disease cases. To gain definitive information about this group would require a second screening.

\section{CONCLUSION}

Potential coeliac disease requires follow-up because of the high risk of conversion to disease with enteropathy. The risk of receiving a clinical diagnosis within 5 years from a screening is very low indicating that a possible repeated screening can wait longer than this.

Acknowledgements We would like to thank Carina Lagergvist for her work with the study biobank, Susanne Walther for the administration of the National Swedish Childhood Coeliac Disease Register, all collaborators within the ETICS study and of course all participating children and families.

Contributors $\mathrm{Al}, \mathrm{AC}, \mathrm{AM}, \mathrm{FN}, \mathrm{LS}$ and $\mathrm{OS}$ were responsible for the conceptualisation of the study. FN performed the statistical analyses. OS, FN and AM wrote the manuscript. OS, LH, MvdP, LS and CW were responsible for data collection. All authors contributed to interpretation of study findings and revision of the manuscript and have approved the submitted version of the manuscript. OS accepts full responsibility for the finished work, had access to the data, and controlled the decision to publish.

Funding Open access funding was provided by Umeå University. This original study was funded by the European Union-supported project FP6-2005-FOOD4B-36383-PREVENTCD, the Swedish Research Council (grants 521-2004- 7093 and 521-2007-2953), the Swedish Research Council for Environment, Agricultural Sciences and Spatial Planning (grants 222-2004-1918 and 222-2007-1394) and the Swedish Council for Working Life and Social Research (grant 2005-0802). We also received funding from Västerbotten County Council for this specific follow-up (grant RV-238151).

\section{Competing interests None declared.}

\section{Patient consent for publication Not applicable.}

Ethics approval This study involves human participants and was approved by the Ethical Review Board of Umeå University, Umeå, Sweden (2004-156 and 20206257). Participants gave informed consent to participate in the study before taking part.

Provenance and peer review Not commissioned; externally peer reviewed.

Data availability statement Data are available on reasonable request. All data included in this study are available after request and discussion with the corresponding author.

Supplemental material This content has been supplied by the author(s). It has not been vetted by BMJ Publishing Group Limited (BMJ) and may not have been peer-reviewed. Any opinions or recommendations discussed are solely those of the author(s) and are not endorsed by BMJ. BMJ disclaims all liability and responsibility arising from any reliance placed on the content. Where the content includes any translated material, BMJ does not warrant the accuracy and reliability of the translations (including but not limited to local regulations, clinical guidelines, 
terminology, drug names and drug dosages), and is not responsible for any error and/or omissions arising from translation and adaptation or otherwise.

Open access This is an open access article distributed in accordance with the Creative Commons Attribution 4.0 Unported (CC BY 4.0) license, which permits others to copy, redistribute, remix, transform and build upon this work for any purpose, provided the original work is properly cited, a link to the licence is given, and indication of whether changes were made. See: https://creativecommons.org/ licenses/by/4.0/.

\section{ORCID iDs}

Olof Sandström http://orcid.org/0000-0003-0899-0531

Anna Myléus http://orcid.org/0000-0003-2478-9598

\section{REFERENCES}

1 Lebwohl B, Sanders DS, Green PHR. Coeliac disease. Lancet 2018;391:70-81.

2 Ravikumara M, Nootigattu VKT, Sandhu BK. Ninety percent of celiac disease is being missed. J Pediatr Gastroenterol Nutr 2007;45:497-9.

3 Bingley PJ, Williams AJK, Norcross AJ, et al. Undiagnosed coeliac disease at age seven: population based prospective birth cohort study. BMJ 2004;328:322-3.

4 Nenna R, Tiberti C, Petrarca L, et al. The celiac iceberg: characterization of the disease in primary schoolchildren. J Pediatr Gastroenterol Nutr 2013;56:416-21.

5 Rubio-Tapia A, Ludvigsson JF, Brantner TL, et al. The prevalence of celiac disease in the United States. Am I Gastroenterol 2012;107:1538-44. quiz 37, 45.

6 Ivarsson A, Myléus A, Norström F, et al. Prevalence of childhood celiac disease and changes in infant feeding. Pediatrics 2013;131:e687-94.

7 Rosén A, Sandström 0, Carlsson A, et al. Usefulness of symptoms to screen for celiac disease. Pediatrics 2014;133:211-8.

8 Lebwohl B, Green PHR, Söderling J, et al. Association between celiac disease and mortality risk in a Swedish population. JAMA 2020;323:1277-85.

9 Kårhus LL, Skaaby T, Petersen J, et al. Long-Term consequences of undiagnosed celiac seropositivity. Am J Gastroenterol 2020;115:1681-8.

10 Turner J, Pellerin G, Mager D. Prevalence of metabolic bone disease in children with celiac disease is independent of symptoms at diagnosis. J Pediatr Gastroenterol Nutr 2009:49:589-93.
11 Troncone R, Ivarsson A, Szajewska H, et al. Review article: future research on coeliac disease - a position report from the European multistakeholder platform on coeliac disease (CDEUSSA). Aliment Pharmacol Ther 2008;27:1030-43.

12 Vilppula A, Kaukinen K, Luostarinen L, et al. Increasing prevalence and high incidence of celiac disease in elderly people: a population-based study. BMC Gastroenterol 2009;9:49.

13 Myléus A, Ivarsson A, Webb C, et al. Celiac disease revealed in 3\% of Swedish 12-year-olds born during an epidemic. J Pediatr Gastroenterol Nutr 2009;49:170-6.

14 Sandström O, Rosén A, Lagerqvist C, et al. Transglutaminase IgA antibodies in a celiac disease mass screening and the role of HLA-DQ genotyping and endomysial antibodies in sequential testing. J Pediatr Gastroenterol Nutr 2013;57:472-6.

15 Ivarsson A, Persson LA, Nyström L, et al. Epidemic of coeliac disease in Swedish children. Acta Paediatr 2000;89:165-71.

16 Namatovu F, Olsson C, Lindkvist M, et al. Maternal and perinatal conditions and the risk of developing celiac disease during childhood. BMC Pediatr 2016;16:77.

17 Aggarwal S, Lebwohl B, Green PHR. Screening for celiac disease in average-risk and high-risk populations. Therap Adv Gastroenterol 2012;5:37-47.

18 Chou R, Blazina I, Bougatsos C. U.S. preventive services Task force evidence syntheses, formerly systematic evidence reviews. screening for celiac disease: a systematic review for the US preventive services Task force. Rockville (MD): Agency for Healthcare Research and Quality (US), 2017.

19 Webb C, Norström F, Myléus A, et al. Celiac disease can be predicted by high levels of anti-tissue transglutaminase antibodies in population-based screening. J Pediatr Gastroenterol Nutr 2015;60:787-91.

20 Norström F, Myléus A, Nordyke K, et al. Is mass screening for coeliac disease a wise use of resources? A health economic evaluation. BMC Gastroenterol 2021;21:159.

21 Johansson K, Norström F, Nordyke K, et al. Celiac dietary adherence test simplifies determining adherence to a gluten-free diet in Swedish adolescents. J Pediatr Gastroenterol Nutr 2019;69:575-80.

22 Volta U, Caio G, Giancola F, et al. Features and progression of potential celiac disease in adults. Clin Gastroenterol Hepatol 2016;14:686-93.

23 Auricchio R, Mandile R, Del Vecchio MR, et al. Progression of celiac disease in children with antibodies against tissue transglutaminase and normal duodenal architecture. Gastroenterology 2019;157:413-20. 